Usage, Usability, and Utility of 3D City Models, 02010 (2012)

DOI: $10.1051 / 3 \mathrm{u} 3 \mathrm{~d} / 201202010$

(C) Owned by the authors, published by EDP Sciences, 2012

\title{
Towards a typology of spatial relations and properties for urban applications
}

\author{
B. Bucher ${ }^{1}$, G. Falquet ${ }^{2}$, E. Clementini ${ }^{3}$ and M. Sester ${ }^{2}$ \\ ${ }^{1}$ IGN, COGIT, 73 avenue de Paris, Université Paris-Est, 94160 Saint Mandé, France \\ ${ }^{2}$ Université de Genève, Centre universitaire d'informatique, 7 route de Drize, \\ 1227 Carouge, Suisse \\ ${ }^{3}$ University of L'Aquila, Dept. of Industrial and Information Engineering and Economics, v. G. \\ Gronchi 18, 67100 L'Aquila, Italy \\ ${ }^{4}$ Institut für Kartographie und Geoinformatik (IKG), Leibniz Universität, Hannover, Germany
}

\begin{abstract}
Relations that occur between features located in space-like the fact that a street is surrounded by very high buildings, that an airport is close to a city- as well as spatial properties of features-like the height and width of a door- play an important role for many urban applications. Digital models of cities can assist in the evaluation of these relations and properties either through visualisation or through computation, mainly based on geometrical information. Hence, considering the objective of explaining to potential users of these city models what useful information they can derive from these data and how, a possible way to address this objective lies in the usage of a pivot model composed of relevant spatial properties and relations, connected to information meaningful to the user and connected to the possible computation of them on available data. This paper firstly sets the ground for a typology of such relevant relations and properties that are shared by different applications and that can be derived/approximated from existing data. It then proposes a model to describe these properties and relations and connect them to their possible computation based on data (2D or 3D). An important aspect of this model is to distinguish between a conceptual layer where relations occur between "real world" features and an implementation layer where they are calculated based on database features and geometries.
\end{abstract}

\section{INTRODUCTION}

There is large variety of applications for city models like for instance land planning, air pollution simulating or training professionals to face situation that cannot be replicated in reality. The accessibility of scientists or users from these domains to the knowledge of how to use these city models is an important stake. It is important on the one hand to ensure an optimal usage of the potential information that can be derived from these data - which requiresan expertness these users possibly don't have and possibly, which is worse aren't aware of. It is also important on the other hand to somehow include the users in the identification of data they need, in order to assess correctly the value of the different data and to fund their acquisition (or not...).

A key assumption in this paper is that there seems to be a layer of relations and properties that meet the needs of most applications. Hence, giving users access to these relations and properties and to the identification of data and tools that will support their evaluation would be a major contribution to our field as well as identifying the contribution of 3D to their derivation or visualisation would be a major contribution to the usage of 3D Citymodels. Typically, pedestrian navigation and orientation can be much enhanced with the analysis of projective relations in a 3D model to support the generation of expressions that are meaningful to users that include relations like "opposite of" [1]. Civil engineering

This is an Open Access article distributed under the terms of the Creative Commons Attribution License 2.0, which permits unrestricted use, distribution, and reproduction in any medium, provided the original work is properly cited. 
applications heavily use topological relations, urban rules are expressed with intervisibility and distance criteria [2, 3]. Research on data integrity use relations as integrity constraints [4]. They have been investigated for 3D as well, focusing on constraints for local climate [5]. Researchers studying urban dynamics focus on relations between cities or between objects within cities like hierarchies and networks[6]. In integration also, spatial relations and properties are useful inputs like in the work of [7] who identify shape and accesses patterns of buildings helps to assess the building functions [7].

The work presented in this paper has been performed in the context of the COST TU0801 action. The aim of this action is precisely to study the semantic enrichment of 3D city models. The action addresses issues such as determining the more relevant enrichment directions; finding efficient enrichment techniques (particularly techniques based on ontologies); and assessing the usability of enriched 3D city models.

Our general objective is to propose a model for users to specify the relations or properties they are interested in and to evaluate how a representation of these properties and relations can be computed from available data based on available operations. For instance, if a user is interested by the distance between individual buildings, he needs data where the individual buildings are not merged into blocks and he can use 2D data. If he needs the distance between windows, he will needs 3D data with a specific LOD. Our model should integrate a shared typology of spatial relations and properties that are important in city models and existing tools and literature. It is due to evolve based on inputs either from scientists working on geocomputation or from users of citymodels. A long term objective of this work is to facilitate discussions and exchange between the various communities dealing with spatial relations and properties in cities, either from the application part or from the geocomputation part. Indeed, as identified in [8], spatial relations have been studied in several scientific domains like computer science, linguistics, philosophy and psychology and within the very domain of computer science.

\section{EXISTING SPATIAL PROPERTIES AND RELATIONS RELEVANT TO THE MANAGEMENT OF SEMANTICS IN CITY MODELS}

This section reviews existing relations and properties that are mentioned or used by authors in diverse works somehow related to cities and to semantics. Semantics here refers to a relationship between data and our experience of the real world.

City abstract features have been studied in the context of urbanism to represent urban rules and important building properties like exposure to sun, intervisibility, and minimum distance. [3] propose and implement a model to represent urban rules and to automatically detect project inconsistencies with respect to these rules. Evaluating a 3D relation may be based on 2D operators but may also necessitate 3D operators like 3D intersection. [2] propose an ontology to represent buildings as arrangements of functional units. They propose a typology of buildings including offices, logistic centre, industrial building, warehouse, researchcentre. Buildings properties are used as variables to adapt an ontology item to a specific case: width, depth and rise. Other properties are different size properties like the ratio between floor gross surface and open surface, construction cost, land value, solar availability, minimal distance (between buildings, from edge). Structures of buildings are described as sets of elementary components and relations between these components. Besides part_of and related_to relation they use an ontology of relations from the architectural domain including topological, directional and proximity relations.

In the domain of sketch maps, landmarks for pedestrian navigation and qualitative geography, the authors identify relevant properties and relations for someone to locate himself otherwise than with coordinates in space $[9,10]$. These works concentrate on identifying relations and properties that can be perceived and that are discriminating, typically a property will be a comparative propertyrelative to a context "the highest building in the neighbourhood". They also focus on how people express these relations, which include vagueness. Finally, some relations are relative to a user point of view like "behind"[1]. 
In 3D, additional relations are relevant: besides directional relations (north, west...) also vertical relations (above, below) are used [11]. Extension of 2D relations to 3D has been especially studied by [12] in the context of virtual environments. He proposes a comprehensive language, based on UMLOCL object constraint language, to define spatial constraints on objects in virtual environments. The core of this extension is a set of spatial relations that may appear within constraint expressions. The available relations are:

- the eight classical topological relations,

- five ternary projective relations in orthographic view (a 2D projection of the scene on one of the $\mathrm{X}=0, \mathrm{Y}=0$, or $\mathrm{Z}=0$ planes): before, between, after, right side, left side

- four ternary projective relations in immersive view: before, between, after, aside

- four quaternary projective relations: inside, outside, above, below

- 27 directional relations from a first-person perspective (avatar view): front, left, above, front-left, front-left-above, etc.

- 27 directional relations from a third-person perspective

- distance function (relative to the bounding box of an object).

The semantics of these relations is based on geometrical approximations of the spatial objects with multiple levels of details. To take into account the imprecise representation of objects the semantics of topological relations is defined on "thick boundary objects".

In interoperability, people study what kind of model is needed to describe a city model. [13] focus on a taxonomy of features to enrich terrain model with more information needed by applications. A specific property in their model is that of level of detail of objects and composition relations. [14] propose a meta-model to describe cities. Their proposal firstly models the urban space through empty space and filled-in/occupied space and interfaces between both. As compared to CityGML, this model definitely puts a highlight on topological relations and more precisely the ability to go from one place to another. Secondly, their model includes new kinds of features like juridical features (people, organisms, etc.) and abstract features (cadastre) which in turn will lead to including new kind of relation in a model like "own", "is applicable on the jurisdiction area", "sits in". Last, they distinguish properties of real world features and properties of database objects.

Other works enrich the data with explicit semantic properties based on geometries. Shapes, symmetries, repetition are important to classify buildings and networks. Methods for detecting regularities and/symmetries are important as buildings do exhibit such a structure. [15] find regularities in 3D models starting from characteristic points, defined by the curvatures of the model's surface. Hypotheses of point pairs corresponding to symmetries are found using the RANSAC principle. [16] finds symmetries in 2D building ground plans using string matching. In [17] also, an important property is regularity or symmetry. [18] find repetitive structures based on local similarity sets gained with the approach of [15], and extend them to uniform grids. Recognition of repetitive structures in road networks has been investigated by [19]. Another possibility is to use formal grammars as a structural representation of facades, as has been used for the interpretation of building facades [20] and for building reconstruction[21]. In [7] composition relations are analysed based on access to the street network and functions are assessed based on these too as well as on shape of sets of buildings. In [22] the case study is flood management and important properties and relations are relative to the shape of the ground: being planar, being $n$ meters above the level of the river. In a similar domain, [23] who concentrates on preserving a 3D topology relation: the hydrography network outflows in the valleys of the relief.

Last, an interesting process in our context is the generalisation of urban models in 2D and 3D. A generalisation process aims either at modifying objects to support the drawing process or at changing a level of details of a representation. Authors identify important information that must be preserved during the data transformation process even if this information is not explicit in the original and final data. This information corresponds, in our view, to the definition of valuable semantics. In the domain 
Usage, Usability, and Utility of 3D City Models

\begin{tabular}{|c|c|}
\hline \multicolumn{2}{|c|}{ Properties and relations strongly connected to our experience of reality } \\
\hline $\begin{array}{l}\text { - } \\
\text { - } \quad \text { shape (width, depth, rise, pattern) of features } \\
\text { or of feature sets } \\
\text { - } \quad \text { salience (the highest building around) } \\
\text { - } \\
\text { - } \text { size, level of detail } \\
\text { belonging to a group, being isolated }\end{array}$ & 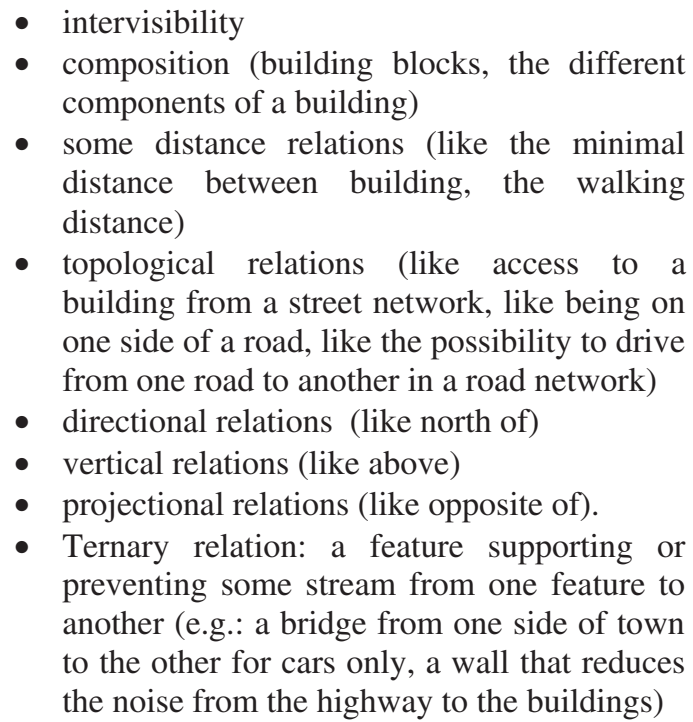 \\
\hline
\end{tabular}

\begin{tabular}{ll}
\hline \multicolumn{3}{|c|}{ Properties and relations strongly connected to computation } \\
\begin{tabular}{lll}
\hline distribution, proportions, regularities, & distance relations (like the minimal distance \\
symmetries & between building, the walking distance) \\
- convexity, etc. & topological relations (RCC8) \\
- resolution, granularity & - directional relations (like aligned buildings)
\end{tabular}
\end{tabular}

Figure 1. Some relations and properties are much connected to semantics and others much connected to computation.

of generalisation, a general property attached to a representation is the required level of detail, e.g. the scale of a map [24]. Objects also have some inherent scale property. Last, the same object may have several representations at different levels of details. This exists in CityGML, where there are several properties connecting an object to its geometry corresponding to five different levels of detail important in city models (e.g. lod0Geometry, lod 1 Geometry, etc.). In a cartographic generalisation process a very important property attached to a representation is that of minimal dimension and minimal distance so that a detail is legible and two features can be distinguished. A typology of constraints on a process is proposed by [25]: topology, position/orientation, shape, pattern, distribution/statistic. Some constraints explicitly refer to relations (topology, orientation) or properties (shape) and others don't but are expressed, in the computing model, as relations and properties. To summarize, the main relations in generalisation are: topology (between buildings and streets), relative orientation, distance, alignment, density, belonging to a group, being decomposed into (e.g. a city is decomposed into a street network and building blocks, a building block is decomposed into buildings). The main properties are: orientation, isolation, shape, size, and granularity. There are several approaches for 3D-generalization; many of them extend existing 2D approaches to 3D. As generalisation requires a large set of operators (to characterise and transform), generalisation of 3D data will progress with the availability of 3D operators like intersection [26].

To summarize this section, there is a set of properties and relations used in several urban applications that need geographical data to be visualised or computed. Several aspects must be highlighted. Firstly, some spatial properties or spatial relations are closer to our experience of the reality and others are closer to the expertness of geocomputation people (see figure 1). A model that maps relations and 
properties that belong to the vocabulary of users of city models with relations and properties that can be computed based on available data is needed to enhance the use of existing city data. Secondly, labels can be ambiguous like "topological relations" which refers to different things (see figure 1). Linguistics is useful to describe spatial properties and relations in user application model. Last, granularity and vagueness of these properties and relations are to be handled both from the user side and from the computation side. A user describing that a house touches another may mean that the house is actually very close to the other but possibly don't touch it actually. The distance between buildings computed on large scale 2D data comes with some uncertainty due to resolutions and errors rate.

\section{FORMAL REPRESENTATION OF THESE RELATIONS AND PROPERTIES AND THEIR COMPUTATION}

The contribution aimed at in this paper is proposing an ontology in the sense of a shared (non ambiguous) model with a goal for exhaustiveness. This ontology should support an application designer making wise choices in terms of data (2D or 3D) and algorithms selection. It is based on the three levels described in section 3.1 .

\subsection{Application, computation and geometric levels}

Spatial relations and properties can be described based on three different representation levels.

- The application level is close to our experience of reality and to the concepts handled in applications. It is required in our proposal to connect to users of citymodels.

- The computation levelis the level of data and operations on data. It is required to index existing models and implemented tools.

- The geometrical level is the abstraction of reality based on mathematics. This level is useful to interconnect the two above levels. It is also useful to index existing mathematical methods.

At the application level, spatial objects and relations are those of a specific application context. Spatial relations at this level heavily depend on various factors, such as the peculiarity of the domain, the implicit vagueness and imprecision of user terms, and the variability of terms in a given country and natural language. The definition of ontology to describe the user level concepts is a research issue. There are various approaches in the literature, for example, in the context of urban information systems [27] or more in general in conceptual modelling [28]. Importantly, thislevel is independent of various geometric representations [29]. In particular, it is independent of the dimension of the embedding space: e.g., modelling how cars interact with roads is independent from the fact that we could use a 2-D spatial representation of cars and roads or a 3-D spatial representation.Let us consider a particular context to give an example: a pocket computer with GPS and wireless communication capabilities is used to give indications about the nature of buildings that are at sight of a mobile user, who is moving with a car in the city. This could be expressed in a query-like formulation as follows: "What is the name of the buildings that are to the right and to the left and ahead of the user position, for which there are no other obstacles in between and they are at reasonable distance?".

Defining relations at the application level may require defining what kind of user will perceive the relations. A dominant role here is taken by various types of frames of reference [12, 30]. Three basic types of frames of reference are distinguished in the literature [31]: intrinsic frames of reference are established on an anchor object that determines the origin of the coordinate system as well as its orientation. Extrinsic frames of reference may also inherit their origin from an anchor object; however, their orientation is determined by external factors such as the direction of motion or by a conventional object used as landmark. Deictic frames of reference involve three objects: a primary object, a reference object, and a point of view. The orientation of the frame of reference is imposed 
Usage, Usability, and Utility of 3D City Models

on the reference object as seen by the point of view. To further describe these three different types of frame of reference, let us consider the following queries: what is the street to my right? What is the building ahead towards north? What is the building to the left of the city hall? If we analyze the queries above we can see that each of them must be interpreted under a different frame of reference: in the first one, the frame of reference is intrinsic due to the fact that it is determined by the orientation of the standing position or moving direction of the user. In the second one, the underlying frame of reference is extrinsic since the conventional "north" direction is used as landmark. The frame of reference being used in the third query is deictic: the reference object is the city hall and the spatial relation of the unknown building is expressed with respect to the point of view of the user.The above examples can be seen as semantic interpretations of underlying projective properties of spatial objects [32].

The geometric level is an abstract representation in mathematical terms of spatial objects and relations. In most models in the literature, spatial objects are represented as abstract point-sets (e.g. [33]) and spatial relations between objects are defined on specific geometric properties: for example, in the 4-intersection model (4IM) [33], topological relations are defined on the empty/nonempty value of the set intersections of the boundary and interior of two objects. The geometric level can be considered the most primitive level for the study of spatial relations, since it allows finding formal definitions. The other two levels are based on the definition of a spatial relation at the geometric level.

At the computational level, spatial objects are represented as spatial data types and spatial relations between objects correspond to spatial operators. In essence, it is the level of spatial relations as supported by a database system. Various proposals of database systems with spatial relations have been developed over the years (e.g. [34-37]). The issues that are considered at the computational level are mainly related to the system performance to compute the relations. The recent trend is that spatial database systems adhere to the specifications of the Open Geospatial Consortium (OGC): systems such as Oracle, IBM DB2, and PostgreSQL base the definition of their spatial data types and operators on the OGC specification for SQL [38]. For example, the following is a definition of an operator for computing a topological relation: Crosses(g1 Geometry, g2 Geometry): Integer. This operator finds a corresponding definition at the geometric level in the "cross" relation of the Calculus-Based method (CBM) [39].

An application level concept can be related to several computations. This can account for multiple representations in geometry, e.g. a river can be represented as a single line or a complex line, or as a two-dimensional region. Therefore, a semantic relationship between two spatial streams (e.g., a river flows into another river) can be modelled with various geometric relationships based on the adopted representations. The road entities can be mapped to a computation in a 2-D geometric representation where they are represented by polylines and the topological relations by existing models, or they can be mapped to a given 3-D geometric representation where they are represented by surfaces and volumes and the topological relations are taken from a 3D set of relations.

\subsection{Proposed formalism}

\subsubsection{Lifting geometrical relations to the application level}

In order to organize these ontologies and connections we propose to use a description logic formalism and to follow a kind of linguistic approach, inspired by [29]. The idea is to consider geometric level relations, such as 'touches', 'collinear', 'closer', and to define their application level counterparts (called city relations). In general, there is no direct and unique correspondence between these levels. For instance "A touches B" has a well-defined meaning as a topological relationship between geometric objects, while it may have several meanings in the application domains, such as for city objects. It may mean "the volume occupied by A touches the volume occupied by B" or "A and B share a common part, such as a wall", or "A and B touch a connecting object, such as a passageway". Therefore, in the proposed ontology of relations, every geometric level relation $R$ corresponds to a generic city relation City $R$ that may have several subrelations corresponding to different meanings. 

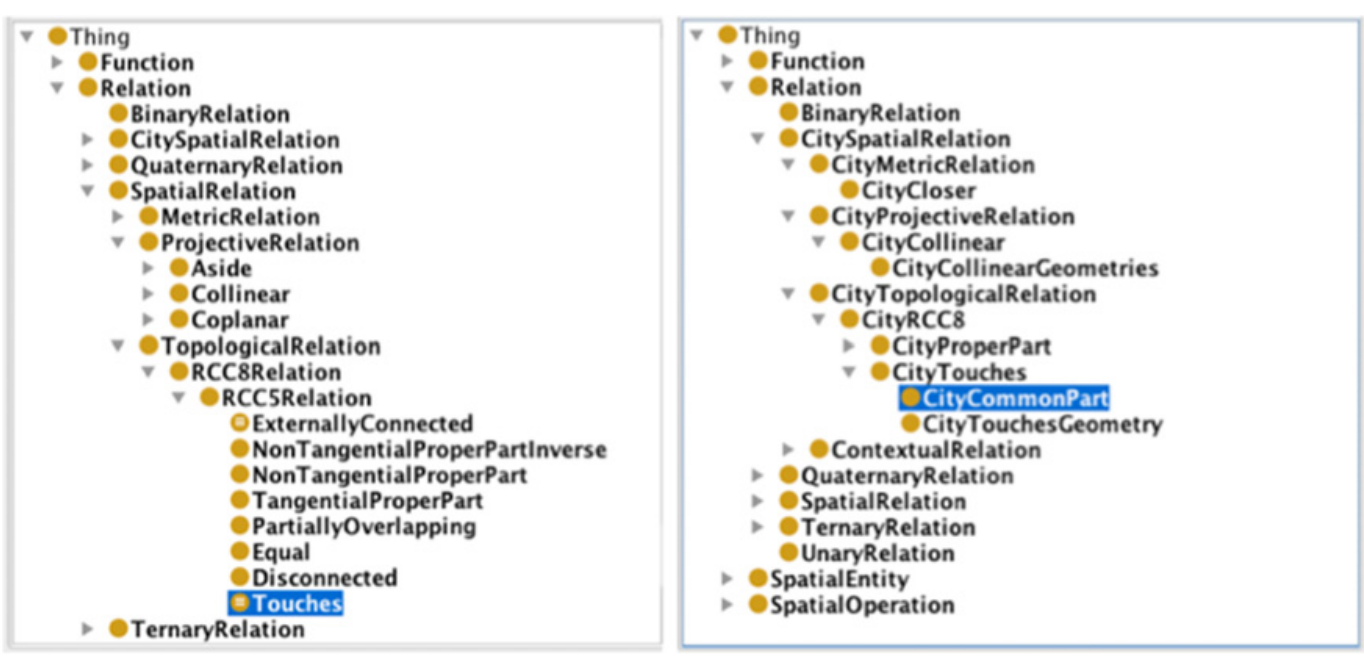

Figure 2. Partial views of the SpatialRelation and CitySpatialRelation subclasses.

Since usual description logics provide only binary relations, we have to represent spatial relations (that can be ternary, quaternary, or more) as objects that belong to subclasses of a general CitySpatialRelation class. Figure 2 shows partial views of the geometric (left) and city (right) spatial relation hierarchies.

Ideally the axioms on spatial relations should be directly transposed to axioms on city relations. This would ensure that the meaning of the city relations is effectively aligned with the meaning of the corresponding spatial relations.

Of course, this approach does not cover all the application-level relations we have identified. Relations such as grouped/isolated, salience, shape, intervisibility, etc. have no direct correspondences at the geometric level. The definition and computation of these relations are generally based on a complex combination of primitive geometric relations.

\subsubsection{Computational context}

In a 3D city model some relations are global, or context dependent. For instance the inter-visibility relation between two points depends on all the objects that belong to the $3 \mathrm{D}$ city model. Topological relations, like A touches B, may also depend on other objects (e.g. in the above-mentioned sense "A touches B if there exists some connecting city object"). Thus, city relations must have a context property that indicates their evaluation context. The context may be the entire set of objects of the 3D city model, or a subset thereof, or a more complex structure.

For instance, the context of some projective relations includes a frame of reference (as mentioned in 3.1). For other projective relations such as intervisibility, the context is the set of all non-transparent solid objects of the model.

The context of a metric relation is a metric space, which is not necessarily the 3D Euclidian space. For instance, the closer relation for pedestrians uses a distance function defined on the union of all the pedestrian traffic areas of the city model.

Complex relations such as isolated/grouped also depend on some distance function on a category of city objects (e.g. a building $x$ is considered as isolated if there is no other building $y$ such that dist $(x, y)$ $<d)$. For a complex relation such as salience, the context comprises a user-specific prominence function (based on height, size, shape, brightness, category, .etc.) 
Usage, Usability, and Utility of 3D City Models

In the ontology the computational context of a city relation is provided by

- a context property that has subproperties such as prominenceFunction, space, frameOfReference, etc.

- axioms that specify the possible values of the context property

- (for complex relations) a baseRelation property that links this relation to the basic relations used in its definition.

For example, the axiom

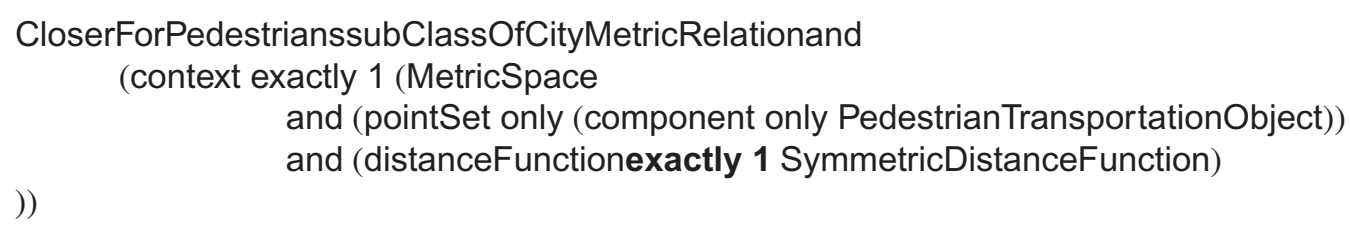

specifices that the CloserForPedestrians relation has a context which is a metric space whose pointsetis made of PedestrianTransportationObjects and whose distance function is symmetric (there are not one-way sidewalks).

\subsubsection{Formal semantics}

Description logics are well suited to represent structural properties of relations (their type, parameter types, context, etc., as shown above) but they cannot express the full semantics of these relations. For this purpose we must turn to more expressive constraint languages (such as first order logics or the OCL object constraint language) or to imperative (algorithmic) languages. Then the formal semantics of a relation will be given by an expression whose predicate, function, or set symbols are drawn from the description of the relation. For instance, the following expressions may provide (simplified) semantics for the Intervisibility and Salient relations in first order logics ${ }^{1}$ :

$$
\begin{gathered}
\text { Intervisibility }(x, y) \Leftrightarrow \forall z \in \operatorname{Intervisibility.context~}(\neg \operatorname{Between}(z, x, y)) \\
\text { Salient }(x)<=>\Leftrightarrow \forall \quad z \in \text { Salient.context (Salient.prominence }(x)>\text { Salient.prominence }(y))
\end{gathered}
$$

\subsection{Further use cases of the ontology}

As stated before, this ontology is firstly aimed at supporting knowledge sharing between communities (like users of citymodels and experts in geocomputation). Yet, in this section we consider further use cases that can be designed to exploit this ontology.

First use cases are related to using the ontology to facilitate assisted discovery/retrieval/derivation of relevant data or of relevant information. Assisted selection of data and derivation of relevant relationsis adequate, if knowledge from the application domain is available which describes which relations are relevant. Mining a data set for dominant relations or properties is relevant when the user when to acquire knowledge about a city based on existing data about this city, in other words to look for dominant structures or relations in these data sets. In this case, all possible relations are evaluated and the most frequent or important in an information theoretic sense are selected and presented to the user.

Other use cases are related to documenting vagueness and to quality and integration. Vagueness in relations may result from vagueness in the definition of spatial objects (e.g. a regions such as "downtown" does not have a crisp border) or in the definition of the spatial relations themselves (e.g. "close to" or "left of" are defined up to a certain degree). Moreover, the computational representation

\footnotetext{
${ }^{1}$ Such expressions can be represented in a description logics ontology (either as text strings or as instances of a LogicalExpression class) but no automated reasoning can be carried out on them.
} 
of geometrically crisp objects is generally imprecise at the computational level (due to limited precision in number representation). Therefore the evaluation of user relations must incorporate a mechanism to handle imprecision and vagueness. The relation ontology must explicitly expose this mechanism for each relation evaluation technique. Thus every such technique must be linked to the appropriate concept within a taxonomy of vagueness representation techniques (that will ultimately form a module of the ontology of spatial relations). Besides, we think that based on this ontology, it could be possible to list at the application level relations that should always be preserved. This list could be useful to evaluate them systematically on existing datasets and, provided the documented vagueness, to detected inconsistencies.

\section{CONCLUSION}

The availability of 3D models is rapidly growing. Advanced application will be possible, if advanced explicit properties and relations will be contained in the model. Automatically extracting these properties and relations and use them in an application dependent way has a large potential of research and poses some research challenges. This paper has drafted a list of important relations and properties in city models and an extension of CityGML to handle them. We list relevant relations and properties at the same time in application domains (so far architecture and urbanism) and of specialists of geodata management (DB integrity management, generalisation) to propose an ontology that will relate relevant relations with operations on data to determine them.

Besides pursuing the implementation of this extension, further work will also address the completeness of the model, from the point of view of experts from application domains and from the point of view of specialists in computational geometry.

We can notice that many city semantics are related to streams in the broadest sense of this word (stream of people, car, sun light, view, water, wind, noise, pollution). Other important aspects are aesthetics, symmetries and repetitions revealing man made features. Hence, we see some interesting research perspectives in adding a high level layer in an ontology of spatial relations and properties considering appearance and affordance.

This work has been partly funded by the European Commission under the COST Action TU0801.

\section{References}

[1] Bartie, P., Reitsma, F., Clementini, E., Kingham, S., 2011, Referring Expressions in Location Based Services: The Case of the 'Opposite' Relation. In: Advances in Conceptual Modeling. Recent Developments and New Directions - Er 2011 Workshops - the Fifth International Workshop on Semantic and Conceptual Issues in Gis (Secogis 2011) (Ed. by O. De Troyer, C. Bauzer Medeiros, R. Billen, P. Hallot, A. Simitsis \& H. Van Mingroot), LNCS 6999, 231-240. Springer-Verlag, Berlin Heidelberg

[2] Caneparo, L., Collo, M., di Giannantonio, D., Lombardo, V., Montuori, A., Pensa, S., Oct. 2007. Generating urban morphologies from ontologies. In: 2nd Workshop COST Action C21 - Towntology

[3] Brasebin, M., Perret, J., Haëck, C., Sep. 2011. Towards a 3D geographic information system for the exploration of urban rules: application to the French local urban planning schemes. In: 28th Urban Data Management Symposium (UDMS2011)

[4] Clementini, E., Tarquini, F. (2008): "Spatial relations between classes as integrity constraints", in Transactions in GIS, Vol12, pp.45-58

[5] Xu, D., 2011: Design and Implementation of Constraints for 3D Spatial Database - Using Climate City Campus Database as an Example, MSc thesis, Delft University of Technology, August (2011) 
[6] Bretagnolle, A., Pumain, D., 2010, Simulating urban networks through multiscalar space-time dynamics (Europe and United States, 17th-20th centuries), Urban Studies, vol.47, $\mathrm{n}^{\circ} 13$, pp. 2819-2839

[7] Chaudhry, O., Mackaness, W., Regnauld, N. (2009): "A functional perspective on map generalisation", in Computers, Environment and Urban Systems (CEUS), vol 33, pp. 349-362

[8] Clementini, E., Laurini, R. (2008) : "Un cadre conceptuel pour modéliser les relations spatiales", in Revue des Nouvelles Technologies de l'Information (RNTI), vol. RNTI-E-14, pp. 1-17

[9] Kopczynski, M. (2006). "Efficient spatial queries with sketches". - In: Proceedings of ISPRS Technical Commission II Symposium, Vienna, Austria

[10] Wang, J., Mülligann, C., Schwering, A (2011): “An Empirical Study on Relevant Aspects for Sketch Map Alignment", in proceedings of the 14th AGILE International Conference on Geographic Information Science (AGILE 2011).Utrecht, The Netherlands, LNGC, pp. 497-518

[11] Borrmann, A. \& E. Rank, 2009: Specification and implementation of directional operators in a 3D spatial query language for building information models, in Advanced Engineering Informatics, 23 (2009), 32-44

[12] Trinh, T.-H. (2012). "A constraint-based approach to modelling spatial semantics of virtual environments", $\mathrm{PhD}$ thesis of Université Européenne de Bretagne, Brest, France

[13] Kolbe, T., Gröger, G., Plümer, L. (2005): "CityGML - Interoperable Access to 3D City Models", in: Oosterom, P., Zlatanova, S., Fendel, F. (eds), Proceedings of the International Symposium on Geo-information for Disaster Management, Springer Verlag, Delft

[14] Billen, R., Laplanche, F., Zlatanova, S., Emgard, L., 2008, Vers la génération d'un méta-modèle générique de l'information spatiale 3D urbaine, in Revue XYZ, N¹14, pp. 37-42

[15] Mitra, N.J., Guibas, L., Pauly, M. (2006): "Partial and Approximate Symmetry Detection for 3D Geometry", ACM SIGGRAPH 2006

[16] Haunert, J. A. H., 2011: Detecting Symmetries in Building Footprints by String Matching. In: S. Geertman, W. Reinhardt und F. Toppen (Eds.): Advancing Geoinformation Science for a Changing World - Proc. 14th AGILE International Conference on Geographic Information Science, Lecture Notes in Geoinformation and Cartography, pp. 319-336. Springer, Berlin, Germany

[17] Kieler B. (2007): "A geometry-driven approach for the semantic integration of geodata sets", 23rd International Cartographic Conference (ICC'07), Moscow, Russia

[18] Pauly, M., Mitra, N.J., Wallner, J., Pottmann, H., Guibas, L.J. (2008): "Discovering Structural Regularity in 3D Geometry", ACM Transactions on Graphics (Proceedings of SIGGRAPH)

[19] Heinzle, F., Anders, K.-H. (2007): “Characterising Space via Pattern Recognition Techniques: Identifying Patterns in Road Networks". In: Generalisation of Geographic Information: Cartographic Modelling and Applications, Mackaness, W., Ruas, A., \& Sarjakoski, T. (ed.), published on behalf of the International Cartographic Association by Elsevier, p. 233-253

[20] Ripperda, N., Brenner, C. (2009): "Application of a Formal Grammar to Facade Reconstruction in Semiautomatic and Automatic Environments", Proceedings of 12th AGILE Conference on GIScience, Hannover, Germany

[21] Huang, H., C. Brenner, Sester, M. (2011): "3D Building Roof Reconstruction from Point Clouds via Generative Models", 19th ACM SIGSPATIAL International Conference on Advances in Geographic Information Systems (GIS), p. 16-24

[22] Klien, E. and Lutz, M. (2005). The Role of Spatial Relations in Automating the Semantic Annotation of Geodata. In Cohn, A. and Mark, D., editors, Spatial Information Theory: InternationalConference, COSIT 2005, Ellicottville, NY, USA, September 14-18, 2005, LectureNotes in Computer Science (LNCS), p. 133-148. Springer

[23] Gaffuri J. (2007): "Outflow preservation of the hydrographic network on the relief in map generalisation”, 23rd International Cartographic Conference (ICC'07), 4-10 August, Moscow, Russia 
[24] Mackaness, W., A. (2007): "Understanding geographic space”, in Generalisation of Geographic Information: Cartographic Modelling and Applications, Mackaness and Ruas (eds), Elsevier

[25] Burghardt, D., Schmid, S., Stoter, J., 2007. Investigations on cartographic constraint formalisation. In: 10th ICA Workshop on Generalisation and Multiple Representations

[26] Kada M (2007) Scale-Dependent Simplification of 3D Building Models Based on Cell Decomposition and Primitive Instancing. In: COSIT 2007, p. 222-237

[27] Berdier, C. \& Roussey, C. (2006) Urban Ontologies: The Towntology Prototype towards Case Studies. Workshop of COST Action C21 Towntology - Ontologies for Urban Development: Interfacing Urban Information Systems. Université de Genève, 91-102

[28] Parent, C., Spaccapietra, S., Zimanyi, E. (2006): "Conceptual Modelling for Traditional and Spatio-Temporal Applications - the Mads Approach". Springer, Berlin

[29] Clementini, E. (2010): “Ontological Impedance in 3D Semantic Data Modeling" In: 5th 3d Geoinfo Conference (Ed. by T. H. Kolbe, G. König \& C. Nagel), ISPRS Conference vol. XXXVIII-4, part W15, 97-100. ISPRS, 3-4 November 2010, Berlin, Germany

[30] Clementini, E. (2012) Directional Relations and Frames of Reference. GeoInformatica, doi: 10.1007/s10707-011-0147-2

[31] Retz-Schmidt, G. (1988) Various Views on Spatial Prepositions. AI Magazine, 9, 95-105

[32] Clementini, E. \& Billen, R. (2006) Modeling and Computing Ternary Projective Relations between Regions. IEEE Transactions on Knowledge and Data Engineering, 18, 799-814

[33] Egenhofer, M.J. \& Franzosa, R.D. (1991) Point-Set Topological Spatial Relations. International Journal of Geographical Information Systems, 5, 161-174

[34] Frank, A.U. (1982) Mapquery: Data Base Query Language for Retrieval of Geometric Data and Their Graphical Representation. ACM Computer Graphics, 16, 199-207

[35] Güting, R.H. (1988) Geo-Relational Algebra: A Model and Query Language for Geometric Database Systems. In: Advances in Database Technology - Edbt'88, Proceedings of the International Conference on Extending Database Technology, March 14-18, 1988, Venice, Italy (Ed. by J. W. Schmidt, S. Ceri \& M. Missikoff), LNCS, 303, 506-527. Springer-Verlag, Berlin

[36] Herring, J. (1991) Tigris: A Data Model for an Object-Oriented Geographic Information System. Computers and Geosciences, 18, 443-452

[37] Cardenas, A.F., Ieong, I.T., Taira, R.K., Barker, R. \& Breant, C.M. (1993) The Knowledge-Based Object-Oriented Picquery + Language. IEEE Transactions on Knowledge and Data Engineering, 5, 644-657

[38] OGC Open Geospatial Consortium Inc. (2006) Opengis®Implementation Specification for Geographic Information - Simple Feature Access - Part 2: Sql Option. OGC 06-104r3, 110

[39] Clementini, E., Di Felice, P. \& van Oosterom, P. (1993) A Small Set of Formal Topological Relationships Suitable for End-User Interaction. In: Advances in Spatial Databases - Third International Symposium, Ssd '93 (Ed. by D. Abel \& B. C. Ooi), Lncs, 692, 277-295. SpringerVerlag, Berlin 